\title{
AN EXPERIMENTAL MODEL OF SMALL INTESTINAL SUBMUCOSA AS A GROWING VASCULAR GRAFT
}

Monica C. Robotin-Johnson, FRACS

Paul E. Swanson, MD

David C. Johnson, FRACS

Richard B. Schuessler, PhD

James L. Cox, MD
Objective: The ideal vascular graft for use in children with congenital heart disease should not only be biocompatible and nonthrombogenic and present no infectious risk, but ideally it should grow at the same rate as the recipient. Methods: We have tested autologous small intestine submucosa as a superior vena cava interposition graft in 11 piglets. The grafts were prepared from segments of jejunum, rendered nonthrombogenic by heparin bonding. The superior vena cava from the level of the azygos vein to the superior vena cava-right atrial junction was replaced. Results: One early and 1 late death were not related to the graft material. At 90 days, the weight of the 9 survivors increased by $630 \%$, from a mean of $10.3 \pm 2.0 \mathrm{~kg}$ to a mean of $59.2 \pm 16.7 \mathrm{~kg}(P<.001)$. The grafts increased in circumference by $184 \%$, from a mean of $36.8 \pm 4.4 \mathrm{~mm}$ to a mean of $61.4 \pm 12.1 \mathrm{~mm}(P<.001)$ at late follow-up. Their length increased by $147 \%$, from a mean of $9.9 \pm 2.1 \mathrm{~mm}$ at implantation to a mean of $15.8 \pm 5.5 \mathrm{~mm}$ at explantation $(P=.002)$. At the time of explantation, all 11 grafts were patent and free of thrombus. Cavograms showed no anastomotic stricture or aneurysm formation in 7 of 9 cases. The luminal surface of all grafts was smooth, shiny, and indistinguishable from that of the native cava. Light microscopy showed a loosely textured collagen framework, with a dense capillary network and complete luminal coverage by a single, continuous cell layer displaying the ultrastructural features characteristic of endothelial cells. Conclusion: Small intestine submucosa provides a collagen framework that becomes remodeled, grows, and acquires a nonthrombogenic endothelial lining. This makes it potentially well suited as a cardiovascular substitute in children. (J Thorac Cardiovasc Surg 1998;116:805-11)
$T^{\mathrm{h}}$ he search for an ideal vascular graft, suited to the pediatric population, has so far yielded disappointing results. As is true of any vascular substitute, the graft should be biocompatible, resist degeneration and infection, have good handling properties, and be available readily and in a range of sizes. ${ }^{1}$ In addition, growth potential would be required ideally, to avoid repeat operations as the child grows.

From the Washington University School of Medicine, St Louis, and Georgetown University School of Medicine, Washington, DC.

Read at the Seventy-eighth Annual Meeting of The American Association for Thoracic Surgery, Boston, Mass, May 3-6, 1998.

Received for publication May 8, 1998; revisions requested June 16, 1998; revisions received June 26, 1998; accepted for publication June 29, 1998.

Address for reprints: Monica C. Robotin-Johnson, MD, 272 Whale Beach Road, Whale Beach NSW 2107, Australia.

Copyright $@ 1998$ by Mosby, Inc.

0022-5223/98 $\$ 5.00+0 \quad \mathbf{1 2 / 6 / 9 2 8 0 0}$
The small intestine has been tried as a vascular substitute as far back as 1965, but its efficacy proved very variable in various experimental designs. 2,3 Using only its submucosa and the deep layers of the mucosal layer, Lantz and coworkers ${ }^{4-6}$ obtained superior results when replacing arterial and venous segments in different experimental animals. Using their experience as a starting point, we tested the hypothesis that the submucosa of the small intestine could be used as a template that would enable a vascular graft to grow sufficiently to keep up with an animal's somatic growth.

\footnotetext{
Material and methods

Eleven piglets, weighing between 8 and $14 \mathrm{~kg}$, were used in this study. All animals received humane care, in accordance with the "Guide for the Care and Use of Laboratory Animals" published by the National Institutes of Health (NIH Publication No. 85-23, revised 1985). The research protocol was approved by the Washington University Animal Care Committee.
} 

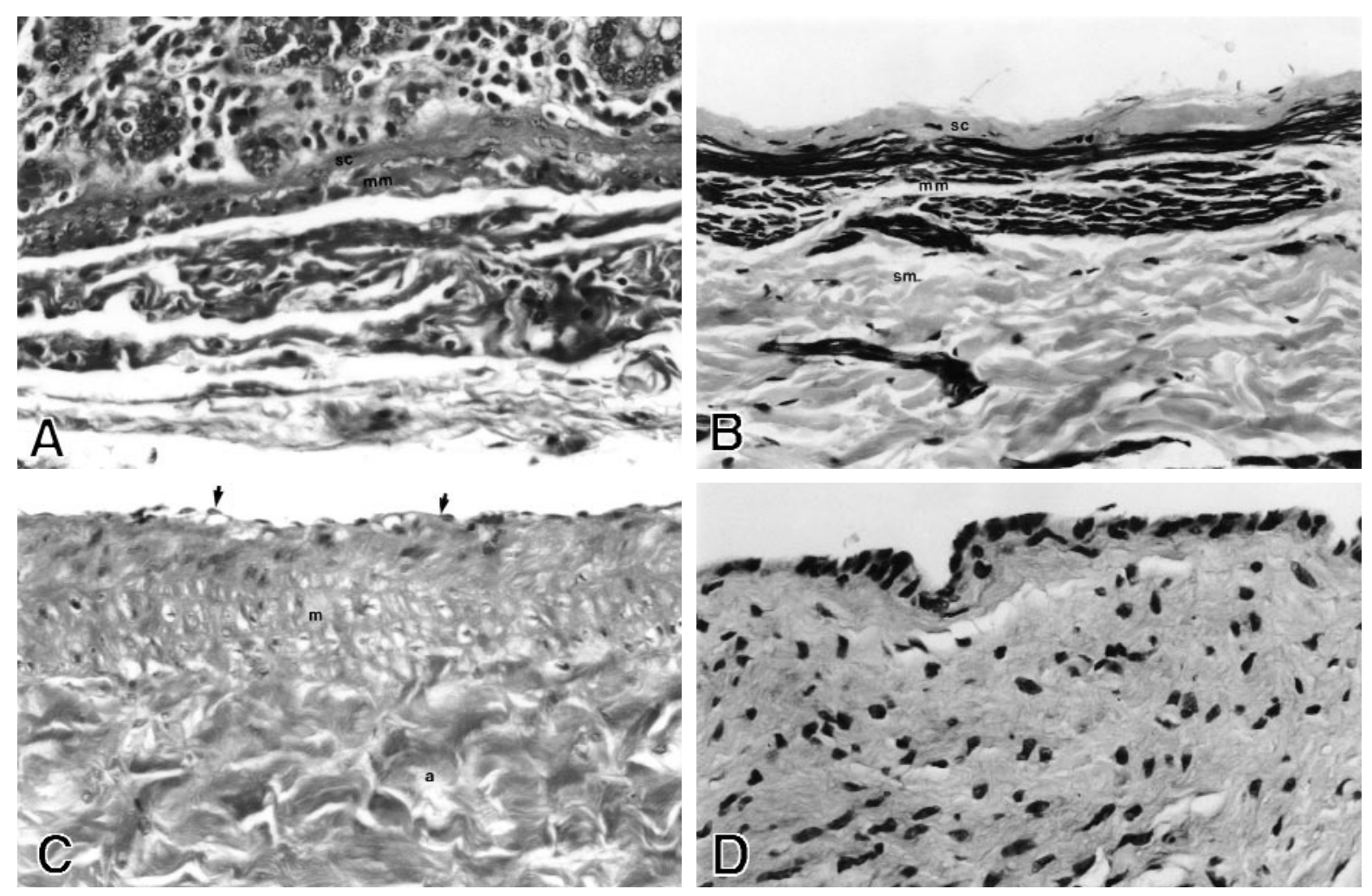

Fig 1. A, Normal small bowel. At high magnification, the interface between submucosa and mucosa is defined by a thin muscularis mucosa $(\mathrm{mm})$ and an overlying delicate paucicellular fibrous band (stratum compactum [ $s c]$ ). The latter elements, together with the submucosa, comprise the graft material illustrated in $\mathbf{B}$. (Hematoxylin and eosin stain; original magnification $\times 500$.) $\mathbf{B}$, The submucosal graft. At a magnification comparable with $\mathbf{A}$, the 3 elements of the graft are evident in a trichrome-stained preparation. The stratum compactum ( $s c$ ), the darkly stained muscularis mucosa $(\mathrm{mm})$, and underlying collagenous submucosa $(\mathrm{sm})$ are demonstrated. Note the vasculature of the submucosa. (Gomori trichromestain; original magnification $\times 500$ ). $\mathbf{C}$, Normal SVC. The cava is lined by an intact endothelium (arrows) that is supported by a media $(m)$ that is composed primarily of smooth muscle. The adventitia $(a)$ is formed of paucicellular, collagenous tissue. (Hematoxylin and eosin stain; original magnification $\times 500$.) $\mathbf{D}$, The submucosal graft ex vivo. A thickened, fibrous, amuscular wall supports an intact, low-cuboidal endothelium. The endothelial layer is taller than in normal cavae (compare with $\mathbf{C}$ ), and the supporting collagenous stroma is more cellular. (Hematoxylin and eosin stain; original magnification $\times 500$ ).

Four pigs, killed at the end of other experiments, with a weight range similar to that of the 11 animals at the end of the study, had measurements of their superior vena cava (SVC) at the same points as the experimental group. They served as a control group.

Surgical procedures and graft preparation. Anesthesia was induced with sodium pentothal $(30 \mathrm{mg} / \mathrm{kg})$ and maintained with isoflurane and intermittent sodium pentothal administration. A catheter was placed in the left femoral artery to monitor blood pressure and obtain blood samples.

Harvest of small intestinal submucosa (SIS). A musclesparing paramedian celiotomy was made, and a 5- to $8-\mathrm{cm}$ long segment of jejunum was excised. Intestinal continuity was reestablished, using a 2-layer anastomotic technique. The abdominal incision was closed in layers.

Preparation of the small intestine submucosa graft. The resected intestinal segment was prepared for use as a vascu- lar graft, according to the technique described by Lantz and coworkers. ${ }^{5}$

In brief, the graft was inverted, and the mucosal layer was removed by gentle abrasion; with the graft in its original orientation, the intestinal serosa and the tunica muscularis were delaminated, with the same technique. This left a translucent graft material, consisting of intestinal submucosa, with attached stratum compactum of the intestinal mucosa and the muscularis mucosae (Fig 1, A). This has been termed small intestinal submucosa (SIS) by previous investigators. ${ }^{5}$ Because the integrity of the stratum compactum appears essential for the graft's thromboresistance, a piece of each graft was sent for histopathologic analysis to confirm the accuracy of graft preparation. ${ }^{6}$

As in our previous experience, most grafts thrombosed early, despite intravenous heparin administration; we used a technique described by Miyata and coworkers, ${ }^{7}$ which enables 


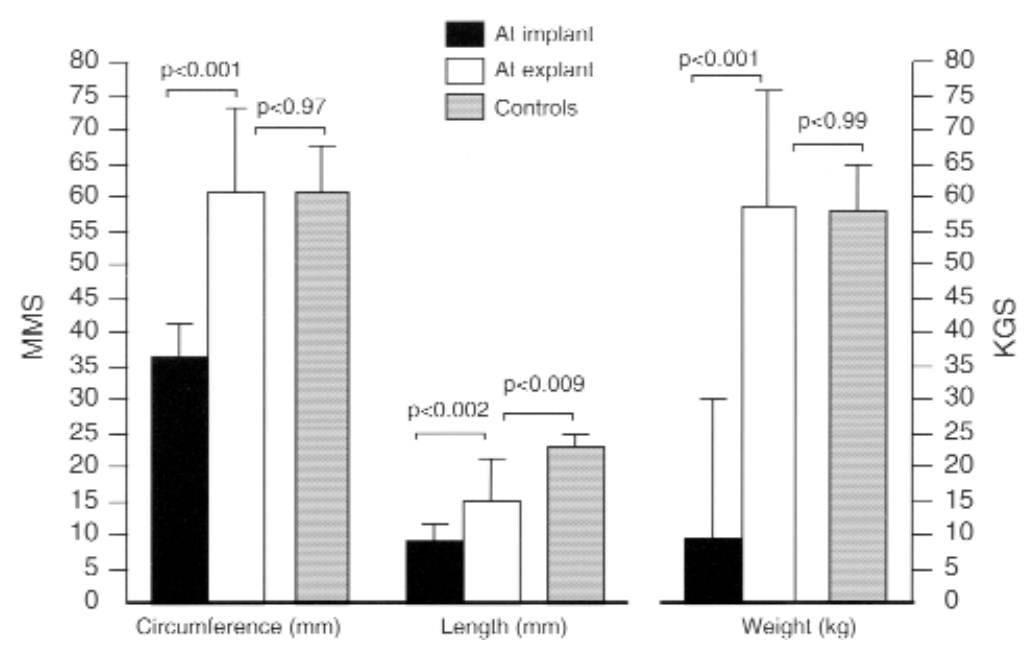

Fig 2. Analysis of measurements of circumference and length of SIS grafts at implantation compared with explantation. The explantation values are compared with control cavae between the azygos vein and the right atrium. The weight of the animals at implantation and explantation and the weight at explantation compared with controls are shown in the far right columns.

heparin to bind to the exposed collagen fibrils and renders the graft non-thrombogenic, while endothelialization occurs.

The graft was soaked in protamine sulfate for 5 minutes, then placed in a $1 \%$ glutaraldehyde solution for 3 minutes, and finally placed in $1 \%$ heparin sodium for 5 minutes. This ensures that the heparin bonds ionically to the protamine, which in turn combines covalently to the collagen, by the action of glutaraldehyde. The graft was then placed in a $10 \%$ neomycin solution for storage.

SVC replacement. A muscle-sparing right thoracotomy through the fourth intercostal space was performed, and the $\mathrm{SVC}$ was dissected free, from the level of the azygos vein to the cavo-atrial junction. The length of the cava and its circumference at these 2 points and the mid-point between these 2 landmarks were measured.

Heparin sodium, 100 units/kg, was administered. Vascular clamps were applied, and the SVC from the level of the azygos vein to the cavo-atrial junction was replaced with SIS, using an end-to-end anastomotic technique. In the first 7 piglets, we used 7-0 polypropylene, and in the last 4 , we used 7-0 polydioxanone suture material.

Although the graft circumference exceeded that of the cava, no longitudinal suture on the SIS was used, but the discrepancy was made up by taking larger bites in the graft than in the native cava.

After de-airing, the clamps were released, and the graft length and maximal circumference were measured. The chest was closed in layers; intercostal nerve blocks, using $0.25 \%$ bupivacaine, were placed, and the animals were allowed to wake up.

Perioperative antibiotic cover was continued for 48 hours, with naxcillin. Except for analgesics, the only other medication the animals received was enoxaparin sodium, 15,000 units, subcutaneously for the first 5 days.
Three months after surgery, the animals were returned to the operating room and anesthetized, by the same protocol. A right internal jugular vein cutdown was performed; heparin sodium 100 units $/ \mathrm{kg}$ was administered, and a large-bore catheter was placed in this vein, for the performance of a cavogram. Iopamiro $(60 \mathrm{~mL})$ contrast material was injected, and biplane angiograms were performed.

A midline sternotomy was performed, and the SVC was dissected free of the surrounding tissues. Measurements of the graft length and circumference at mid-point and the circumference of the native cava were taken.

The animals were then killed, with an injection of sodium pentothal, $120 \mathrm{mg} / \mathrm{kg}$, and potassium chloride, $20 \mathrm{mEq} / \mathrm{kg}$. The graft and the adjacent cava were excised and placed in a buffered formaldehyde solution.

Sections were prepared for light and transmission electron microscopy in a routine fashion, with representative tissues fixed in $10 \%$ buffered formalin and $3 \%$ buffered glutaraldehyde, respectively.

Controls for this study were 4 pigs, in the same weight range as the dead pigs, in which measurements of caval length and diameter were taken, at the same levels.

Data analysis. Comparisons were made with an unpaired (controls versus experimental animals) and a paired (measurements at implantation and explantation for the experimental group) $t$ test, with a Dunn Sidak correction. All data were expressed as a mean \pm standard deviation.

\section{Results}

One piglet died immediately after the operation when, during premature accidental extubation, it became severely hypoxic during attempts at reintubation. The SVC graft was patent. Another piglet died 5 


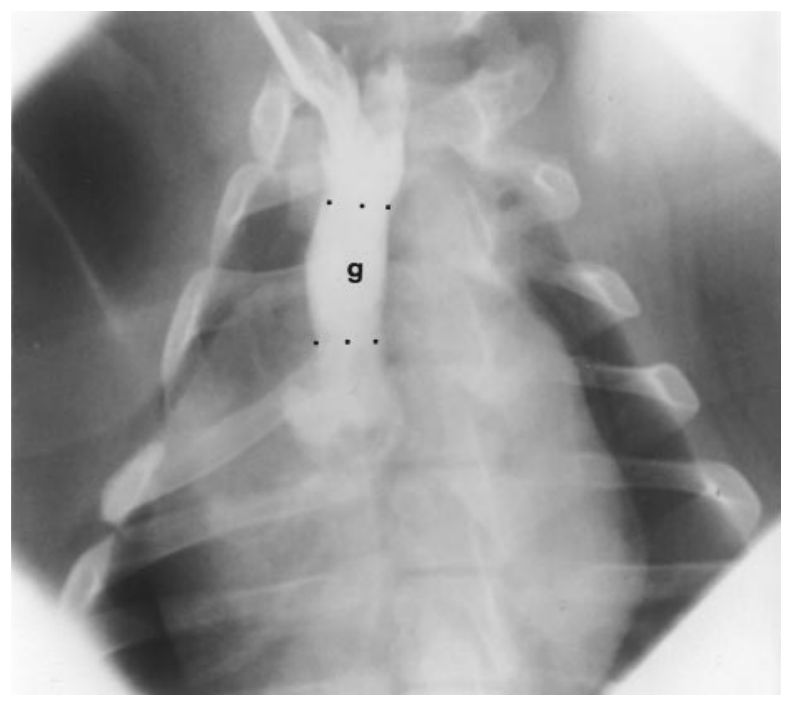

Fig 3. An example of a cavogram shows the graft $(g) 3$ months after implantation, just before explantation. This was unchanged from immediately after SVC replacement, although the animal's weight had increased fivefold.

weeks after operation after a septic episode, characterized by fever, rigors, and prostration, mandating euthanasia. Pathologic examination showed its graft to be patent and the intestinal anastomosis to be completely healed. It had significant hepatomegaly and a large volume of turbid ascitic fluid, growing a Bacillus species. Interestingly, this animal was housed together with another piglet, in which similar symptoms developed, but which recovered after prompt institution of antibiotic cover. However, its SIS graft, although completely incorporated and displaying the same macroscopic and microscopic features as all the others, grew little and displayed an asymmetric central bulge.

Nine piglets survived to the end of the experiment, between 10 and 14 weeks from the original operation. Their weight increased from a mean of $10.3 \pm 2.0 \mathrm{~kg}$ before operation to a mean of $59.2 \pm 16.7 \mathrm{~kg}$ after operation. This compared with a mean weight of $58.5 \pm 6.6$ $\mathrm{kg}$ in controls (Fig 2).

Their grafts were patent by cavogram, with a smooth, slightly fusiform appearance, unchanged from immediately after the SVC replacement (Fig 3).

On measurement, the graft circumference increased by $184 \%$, from a mean circumference of $36.8 \pm 4.4 \mathrm{~mm}$ immediately after operation to a mean circumference of $61.4 \pm 12.1 \mathrm{~mm}$ at late follow-up. This compared with a mean caval circumference of $61.2 \pm 6.4 \mathrm{~mm}$ in the controls. The length of the graft increased by $147 \%$, from a mean of $9.9 \pm 2.1 \mathrm{~mm}$ at implantation to a mean of $15.8 \pm 5.5 \mathrm{~mm}$ at explantation. This compares with a mean of $23.8 \pm 1.2 \mathrm{~mm}$ in the control animals $(P=$ .009). These data are summarized in Fig 2.

Pathologic examination. After preparation, the SIS graft appears macroscopically as a thin, white tube, approximately $0.1-\mathrm{mm}$ thick. Hematoxylin and eosin and van Gieson stains show it to consist of intestinal submucosa, with an intact muscularis mucosa and overlying stratum compactum (Fig 1, $A$ and $B$ ). The intestinal submucosa consists of paucicellular, dense collagen, irregularly arranged, with prominent small venules and arterioles, occasional lymphatic spaces, and rare inflammatory cells (lymphocytes, histiocytes, and mast cells). Small nerves and ganglion cells are also present. The muscularis mucosa is a thin, organized bundle of smooth muscle cells; the stratum compactum is a dense, acellular collagen structure, with a small amount of elastic fibers.

Control SVC tissues exhibit features of normal large veins, including an intact endothelium supported by organized lamina, fibromuscular tissue, and elastic fibers. The media of the SVC consists of organized arrays of smooth muscle cells. Vasa vasorum are generally inconspicuous (Fig 1, C).

The explanted grafts were thicker than the original SVC and densely adherent to the surrounding tissues, from which they were relatively difficult to dissect. The latter compromised efforts to evaluate organization of a neo-adventitia in the graft. The luminal surface of all the grafts appeared shiny, smooth, and free of thrombus or irregularities. The suture lines were discernible as thin, slightly raised ridges in the animals where a non-absorbable suture was used but appeared smooth and flat where an absorbable suture was used.

Microscopically, the grafts were lined by a single, continuous layer of flat or cuboidal endothelial cells, overlying an organized, thick layer of well-vascularized and variably cellular collagen. The endothelial covering was already present in the graft explanted at 5 weeks. A rich capillary network was present across the entire thickness of the graft. The suture lines were inflamed, with a prominent foreign-body reaction. Unlike normal SVC or pre-graft SIS, there was no evidence of an organized vascular media, although smooth muscle cells were focally admixed with fibrous elements near anastomoses and along small intramural vessels. Elastic fibers were haphazardly arranged in the subendothelial tissues (Fig 1, D).

On transmission electron microscopy, the luminal surface was covered by a single layer of cuboidal cells that rested on a continuous lamina. The cells had blunt, interdigitating cell processes with occasional cell junc- 
tions. Pinocytosis was conspicuous on the luminal and basolateral membranes. Weibel-Palade bodies were identified (Fig 4). These ultrastructural features are characteristic of endothelial cells.

\section{Discussion}

A vascular graft with growth properties is ideally required in pediatric patients. This would obviate the need for reoperations, required to match the graft dimensions to those of a growing child, and if completely incorporated, would eliminate the risk of graft infection.

So far, pericardium is the only material coming close to meeting these requirements, although it has been found to be prone to shrinkage, calcification, or alternatively aneurysm formation. ${ }^{8-10}$

Resourceful and inventive cardiovascular surgeons have looked at different alternatives over the years. The use of intestinal wall as a venous conduit was first described by Matsumoto and associates ${ }^{2}$ in 1965 . Since then, other surgeons have also tried it, but the results were quite variable. Lantz and colleagues ${ }^{5}$ developed a new technique, using only the intestinal submucosa as graft material. They reported excellent experimental results using SIS to replace segments of veins and arteries of both large and medium size. ${ }^{4-6}$ The grafts remained patent as long as 5 years after implantation and acquired an endothelial lining similar to the native vessels. They became thicker, matching the thickness of the replaced vessel, and developed a rich vascular supply, which brought along smooth muscle cells and connective tissue, the latter forming an adventitial layer. The authors were able to demonstrate the same good results using SIS xenografts that had been stored for periods of up to 2 weeks. ${ }^{6}$ This led them to presume that the SIS acts as a structural framework on which the cellular organization takes place, leading eventually to the formation of a new graft and resorbtion of the template. This was demonstrated by the same group of investigators by immunohistochemical analysis of the remodeled tissue, suggesting that the SIS material was degraded and resorbed over time. ${ }^{11}$ If the graft becomes replaced by autologous tissue, it would be able to grow at the same rate as the surrounding tissues, which was our working premise. The circumferential growth of the graft exceeded that in length. This may be related to the short segment of cava replaced, making the 2 suture lines relatively close together, with surrounding scarring possibly impairing the growth to some extent, or alternatively it may represent appropriate growth in length for these hemodynamic conditions, given the growth in width of the grafts, which kept the grafts the

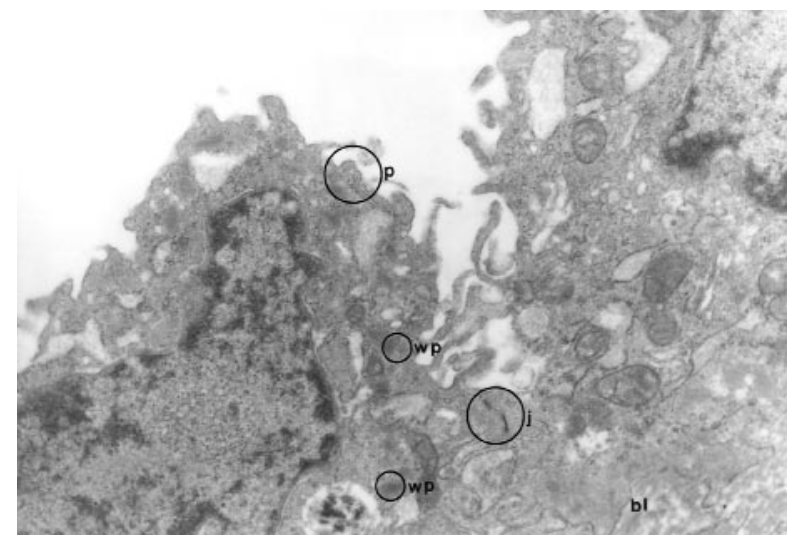

Fig 4. Transmission electron microscopy of graft endothelium. Cuboidal cells are supported along basement membranes by basal lamina $(b l)$. Cell junctions $(j)$ connect apposed lateral cell membranes. Delicate cell processes extend from apicolateral surfaces. Pinocytic vesicles $(p)$ are prominent along all cell surfaces. Occasional Weibel-Palade bodies $(w p)$ can be identified. (Uranyl acetate/lead citrate stain; original magnification $\times 6250$.)

same size as the surrounding cava. A different experiment, replacing a longer segment of vessel, may be able to better answer this question.

If SIS is only a structural framework and considering the fact that the collagen molecule shows little variability among different species, a tissue bank for SIS becomes a possibility, making this material available as an off-the-shelf vascular substitute. The technique of preparation and preservation of the SIS grafts is simple and reproducible. Other investigators ${ }^{12,13}$ have developed other techniques aimed at biocompatibility, such as endothelial cell seeding of Dacron grafts or preparation of an acellular matrix, using a multistep detergentenzymatic process. These techniques are more complex, requiring specialized equipment and many reagents and, in the case of the Dacron-based graft, do not allow any growth to occur.

Lantz and Badylak $^{4,5}$ recommended aggressive anticoagulation, using both aspirin and warfarin (Coumadin) for a period of 8 weeks, time taken by the graft to endothelialize because exposed collagen is very thrombogenic. Even in systemically anticoagulated animals, SIS grafts used as microvascular grafts thrombosed within an hour after implantation. ${ }^{14}$ Our initial experiments in piglets using the original technique described by Lantz and Badylak ${ }^{5}$ led to a high rate of early graft thrombosis. A study of the clotting and lytic mechanisms in man and 7 mammalian species demon- 
strated that overall, the pig had the most reactive blood coagulation mechanisms, clotting the fastest and lysing the slowest. ${ }^{15}$ This made a porcine model ideal for the study of graft patency. We were able to avoid anticoagulation (except for a daily dose of low-molecular heparin for 5 days), by using a technique developed by Miyata and colleagues, ${ }^{7}$ which achieves ionic bonding of heparin to protamine, itself covalently bound to collagen by the action of glutaraldehyde. The ionic bonding permits slow release of the heparin and continuing antithrombogenicity while the graft becomes endothelialized. Obviating the need for systemic anticoagulation represents a major advantage in infants and children, given the difficulties in maintaining the anticoagulation regimen stable and minimizing patient discomfort related to the frequent blood draws required by children receiving warfarin therapy.

The glutaraldehyde also may work to reduce the antigenicity of the collagen, making the graft less likely to engender an immune response from the host. Collagen is a weak antigen and represents an ideal matrix for the ingrowth of host cells. Unlike the conventional glutaraldehyde-treated porcine valves, where collagen is firmly crosslinked by the action of glutaraldehyde and does not allow ingrowth of host cells into the graft, this rapid protamine-glutaraldehyde-heparin soaking technique allows for a weaker covalent binding of the collagen molecule to the glutaraldehyde through the protamine molecule. This probably accounts for the antithrombogenicity of the grafts and the relatively rapid infiltration of the graft by host cells and endothelialization. Even the graft explanted at 5 weeks already displayed an endothelial lining.

Another concern related to the use of prosthetic materials in children is the high rate of calcification observed in implanted homografts, xenografts, and even autologous pericardium..$^{9,16,17}$ We observed no calcification in the grafts, despite using rapidly growing animals, in which the incidence of calcification is expected to be high.

To date, we are aware of no grafts used in the pediatric population that grow with the patient, which represents this graft's greatest advantage over any other grafts currently available.

We are satisfied with the performance of the SIS graft as a venous graft, and we feel that further research is warranted in its use as a heterograft for vascular and cardiac applications to best assess its potential as a cardiovascular substitute in operations such as repair of tetralogy of Fallot, pulmonary artery reconstruction, Fontan procedures, or even valve replacements in infants and children.

\section{REFERENCES}

1. Wright CB, Dunn EJ, Ketterhagen JP, Callard GM, Flege JB. The regulatory environment for vascular grafts. In: Sawyer PN, editor. Modern vascular grafts. New York: McGraw-Hill; 1987. p. 10514.

2. Matsumoto T, Holmes RH, Burdick CO, Heisterkamp CA, O'Connell TJ. The fate of the inverted segment of small bowel used for the replacement of major veins. Surgery 1965;60:73943.

3. Fraser RE, Halseth WL, Johnson B, Paton BC. Experimental replacement of the superior vena cava. Autologous vein, free inverted jejunal grafts, and Dacron grafts treated with GraphiteBenzalkonium-Heparin. Arch Surg 1968;96:378-85.

4. Lantz GC, Badylak SF, Coffey AC, Geddes LA, Sandusky GE. Small intestinal submucosa as a superior cava graft in the dog. J Surg Res 1992;53:175-81.

5. Lantz GC, Badylak SF, Coffey AC, Geddes LA, Blevins WE. Small intestinal submucosa as a small-diameter arterial graft in the dog. J Invest Surg 1990;3:217-27.

6. Lantz GC, Badylak SF, Hiles MC, Coffey AC, Geddes LA, Kokini K, et al. Small intestinal submucosa as a vascular graft: a review. J Invest Surg 1993;6:297-310.

7. Miyata T, Noishiki Y, Matsumae M, Yamane Y. A new method to give antithrombogenicity to biological materials and its successful application to vascular grafts. Trans Am Soc Artif Intern Organs 1983;29:363-5.

8. Mohri H, Barnes RW, Rittenhouse EA, Reichenbach DD, Dillard $\mathrm{DH}$, Merendino KA, et al. Fate of autologous pericardium and Dacron fabric used as substitutes for total atrial septum in growing animals. J Thorac Cardiovasc Surg 1970;4:501-11.

9. Gabbay S, Bortolotti U, Factor S, Shore DF, Frater R. Calcification of implanted xenograft pericardium. J Thorac Cardiovasc Surg 1984;87:782-7.

10. Rosenthal A, Gross RE, Pasternak A. Aneurysms of right ventricular outflow patches. J Thorac Cardiovasc Surg 1972;63:73540.

11. Hiles MC, Badylak SF, Lantz GC, Kokini K, Geddes LA, Morff RJ. Mechanical properties of xenogenic small-intestinal submucosa when used as an aortic graft in the dog. J Biomed Mater Res 1995;29:883-91.

12. Rupnik MA, Hubbard FA, Pratt K, Jarrell BE, Williams SK. Endothelialization of vascular prosthetic surfaces after seeding or sodding with human microvascular endothelial cells. J Vasc Surg 1989;9:788-95.

13. Wilson GJ, Courtman DW, Klement P, Lee JM, Yeger H. Acellular matrix: a biomaterials approach for coronary bypass and heart valve replacement. Ann Thorac Surg 1995;60:S3538.

14. Prevel CD, Eppley BL, McCarthy M, Jackson JR, Voytik SL, Hiles MC, et al. Experimental evaluation of small intestinal submucosa as a microvascular graft material. Microsurgery 1994;15: 586-91.

15. Mason RG, Read MS. Some species differences in fibrinolysis and blood coagulation. J Biomed Mater Res 1971;5:121-8.

16. Cleveland DC, Williams WG, Razzouk AJ, Trusler GA, Rebeyka IM, Duffy L, et al. Failure of cryopreserved homograft valved conduits in the pulmonary circulation. Circulation 1992; 86(suppl):150-3.

17. Milano A, Bortolotti U, Talenti E, Valfre C, Arbustini E, Valente $\mathrm{M}$, et al. Calcific degeneration as the main cause of porcine bioprosthetic valve failure. Am J Cardiol 1984;53:1066-70. 


\section{Discussion}

Dr John H. Kennedy (Cambridge, England). Crucial to the issue of whether these cells revert to a primitive type, as, for example, cultured renal cells do, and then will be truly antithrombogenic, have you really proved that that is true in the long term? I realize that in your fourth slide there was a single cellular layer, and you have led us to believe that that is an endothelial cell layer with all that implies, a failure of rolling and sticking of white corpuscles, for example.

Dr Robotin-Johnson. Thank you for your question. Badylak and Lantz conclusively demonstrated that the single layer of cells lining the luminal surface of SIS grafts is indeed an endothelial layer, by using scanning electron microscopy, as well as immunocytochemistry with factor VIII-related antigen and periodic acid-Schiff/alcian blue staining. To test the lack of thrombogenicity of the grafts, we chose the pig as an experimental model, as it has the most active clotting mechanisms and the less-active lytic mechanisms of all the usual experimental animals. The fact that we did not observe any thrombus formation on any of the grafts seemed to us very encouraging.

Dr Juan C. Chachques (Paris, France). A few years ago, at Broussais Hospital in Paris we made a similar study using autologous pericardium treated with glutaraldehyde. We found similar results at 3 months, but at 1 year there is some stenotic reaction at the level of the sutures. What is the concentration of glutaraldehyde that you use in your study? Because the problem is in the anastomosis of the graft in long-term, which is the material that you use in the anastomosis?

Dr Robotin-Johnson. I would like to answer the second question first. We used 7-0 polypropylene suture (Prolene; Ethicon, Inc, Somerville, NJ) in the first 7 animals. At the end of the experiment, the suture lines were discernible as slightly raised edges, but on histologic examination a foreign-body reaction was quite evident. Subsequently, we changed to 7-0 polydioxanone (PDS; Ethicon) for the last 4 animals and were gratified to observe that the suture lines were no longer discernible with the naked eye, and although on histologic sections the suture material was still present at 10 to 12 weeks, the amount of foreign-body reaction was much reduced.

The method of graft preparation includes only a brief immersion (3 minutes) in a diluted glutaraldehyde solution ( $1 \%$ concentration), which differs from the standard method of fixation for a heart valve, where tanning of the tissue ensures covalent cross-linking of the collagen. Therefore no ingrowth of host cells is possible, and this may be part of the reason that these prosthetic materials fail in the long term, as they never become incorporated into the host. Our preparation ensures a covalent binding of collagen and protamine. Heparin, which is subsequently added, becomes ionically bound to the protamine and available for slow release after implantation, while the graft becomes penetrated by the host cells and endothelialized. As the graft remodels and the ingrowth of the host cells transforms it into a structure of the body's own making, it becomes incorporated and therefore less likely to engender a foreign body reaction and fail.

Dr Fred A. Crawford, Jr (Charleston, SC). Did you follow any of these animals longer than 90 days?

Dr Robotin-Johnson. Not as a part of this study, although we kept an animal alive for 6 months. The graft preparation method was somewhat different, so we did not include it here. His graft was perfectly incorporated and showed evidence of growth. Badylak and Lantz followed up their dogs, in which they replaced carotid arteries with SIS grafts, for up to 5 years. The grafts remained patent and without evidence of intimal hyperplasia. As their method of graft preparation was different, they had to rely on a vigorous anticoagulation protocol, consisting of continuous heparin infusion and warfarin sodium (Coumadin) for the time it took for the grafts to become endothelialized.

Dr Edward D. Verrier (Seattle, Wash). I have 1 final question. The use of vascular grafts in children in the low/ pressure SVC would be pretty unusual. It would be much more common to use it as an arteriovenous graft, like a Blalock shunt, or some type of right ventricular to pulmonary artery graft. Have you used this in a model under higher pressure where the potential for physical forces would be more realistic to what you would see in a clinical situation?

Dr Robotin-Johnson. We used the SIS graft as a venous substitute, as we wanted to answer questions related to the potential thrombogenicity of these grafts, which would be more evident in a low-pressure system, as well as the issue of whether these grafts would grow, rather than simply "stretch" under the influence of arterial pressure. I agree that to broaden the scope of this material, other experiments would be needed and we hope to be able to use them as pulmonary artery substitutes and valve material. 